\title{
Inhibition of eukaryotic elongation factor-2 Confersconfers to tumor suppression by a herbal formulation Huanglian-Jiedu decoction in Humanhuman hepatocellular carcinoma
}

givennameNing Wangsurnamea,

givennameYibin Fengsurname ${ }^{a, b, *}$

yfeng@hku.hk

givennameHor-Yue Tansurname

givennameFan Cheungsurname ${ }^{a}$

givennameMing Hongsurname ${ }^{a}$

givennameLixing Laosurnamea

givennameTadashi Nagamatsusurname ${ }^{c}$

aSchool of Chinese Medicine, Li Ka Shing Faculty of Medicine, The University of Hong Kong, Hong Kong

bShenzhen Institute of Research and Innovation, The University of Hong Kong,Kong, Shenzhen, Shenzhen, China

'Laboratory of Pharmacobiology and Therapeutics, Faculty of Pharmacy, Meijo University, University, Japan

${ }^{*}$ Correspondence to: School of Chinese Medicine, The University of Hong Kong, 1/F, Estate Building, 10 Sassoon Road, Pofkulam, Hong Kong S.A.R. Tel.: +852 2589 $0482 ;$ fax: +852 2168 4259

\section{Abstract}

\section{Ethnopharmacological Relevance}

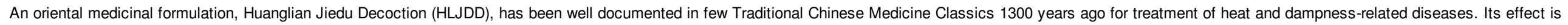

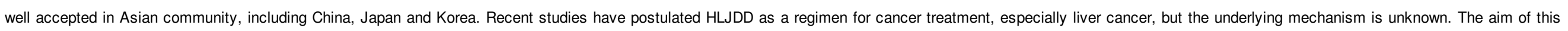
study was teto examine the suppressive effect of HLJDD on the growth of hepatocellular carcinoma (HCC) and its possible underlying mechanism.

\section{Methods}

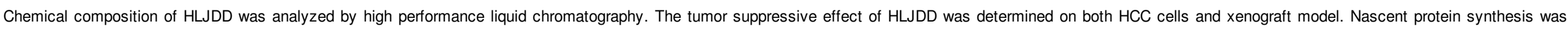
detected with Click-IT protein labeling technology; protein expression was determined by immunoblotting and imunnohistochemical analysis.

\section{Results}

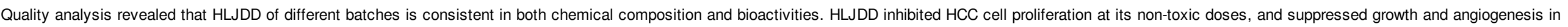

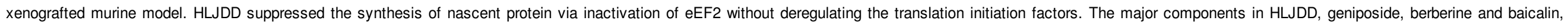

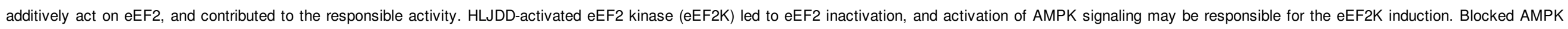

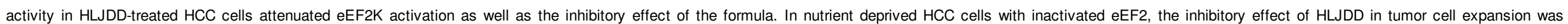
interfered.

Conclusion

Our results indicate that HLJDD has potential in blocking HCC progression with involvement of eEF2 inhibition. 


\section{Introduction}

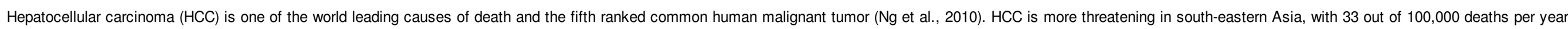

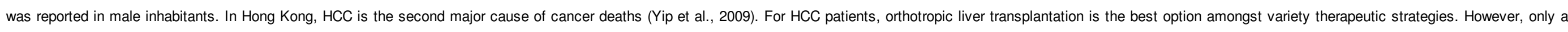

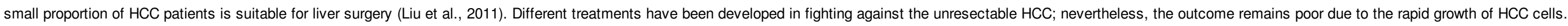

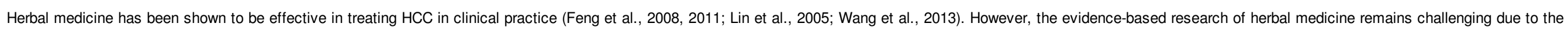

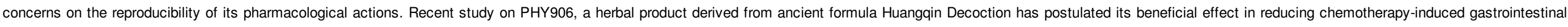

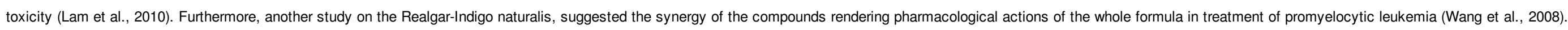

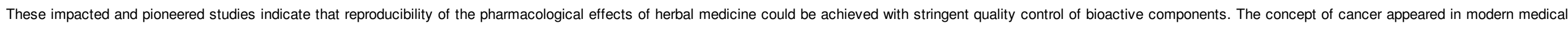

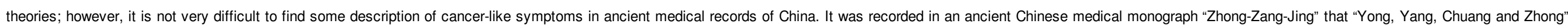

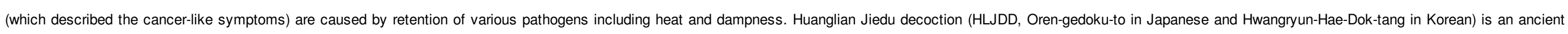

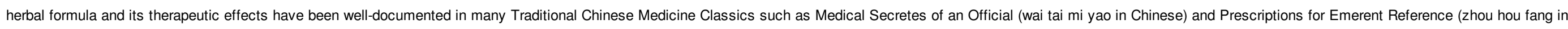

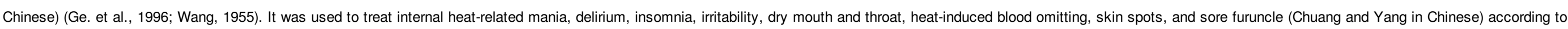

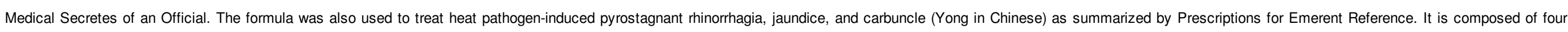

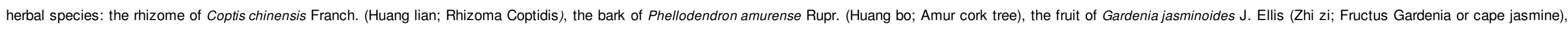

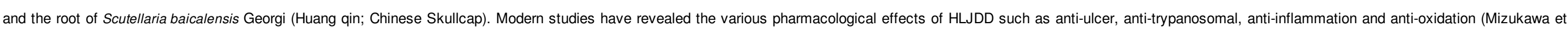

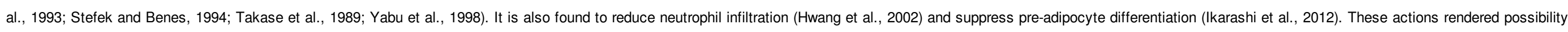

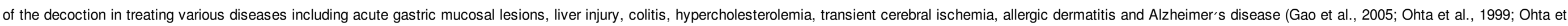

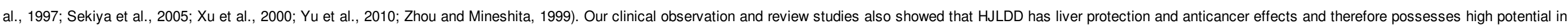

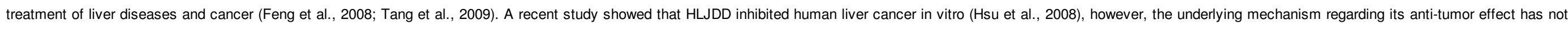
been fully understood.

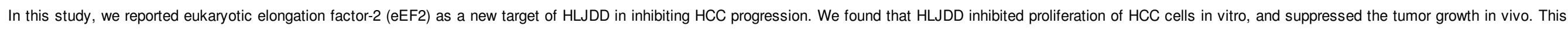

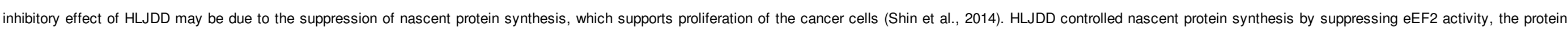

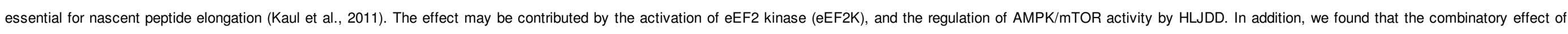

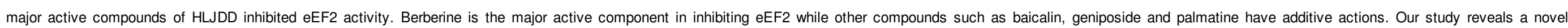
mechanism involved in anti-cancer effect of HLJDD and may indicate the translational potential of the decoction as a complementary agent in HCC treatment.

\section{Material and Methodsmethods}

\subsection{Herbal preparation, chemicals and reagents}

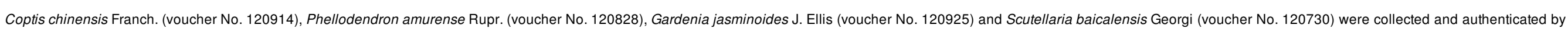

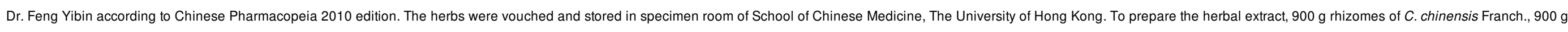

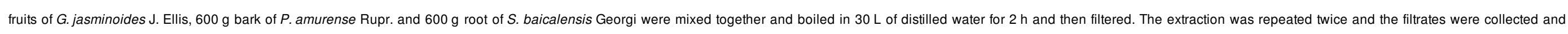
evaporated to dryness. The extract powder was kept in $-20^{\circ} \mathrm{C}$. Three batches of HLJDD were produced. Berberine chloride, palmatine chloride, baicalin and geniposide were purchased from Sigma-Aldrich (USA).

\subsection{Antibodies}

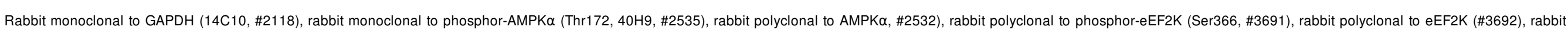

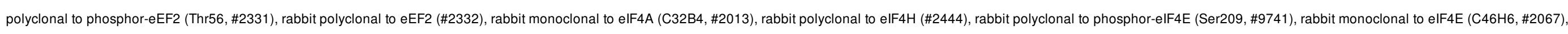

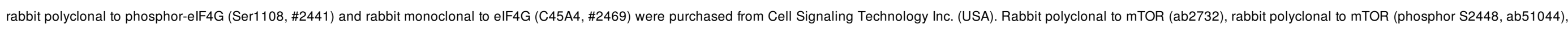




\section{elsevier_JEP_9328}

rabbit polyclonal to Ki67 (ab15580) and rabbit polyclonal to CD31 (ab28364) were purchased from abcam (UK).

\subsection{Chemical analysis}

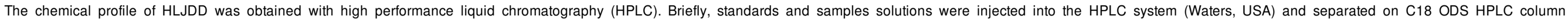

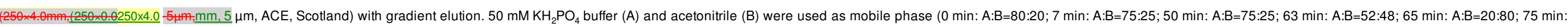

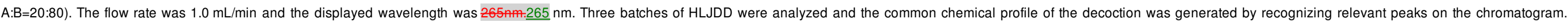
Analysis of any heavy metals and organic pesticides contamination were conducted with methods described in our previous study (Tan et al., 2013).

\subsection{Cells and cell culture}

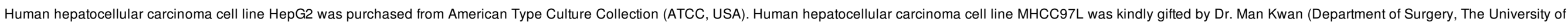
Hong Kong). Cells were maintained in DMEM medium with high glucose and incubated in a humidified atmosphere containing $5 \% \mathrm{CO}_{2}$ at $37^{\circ} \mathrm{C}: 37^{\circ} \mathrm{C}$.

\subsection{Cell viability assay}

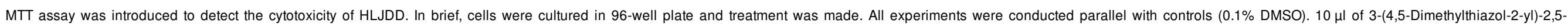

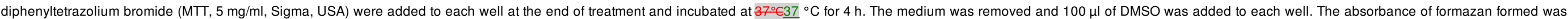
measured at $595 \mathrm{~nm} \underline{595} \mathrm{~nm}$ by Multiskan MS microplate reader (Labsystems, Finland). The percentage of viability was calculated as the following equation: (viable cells)\%=(OD of drug-treated sample/OD of vehicle-treated sample) $\times 100$.

\subsection{Clonogenic assay}

10,000 cells were seeded into 6 -well plate and treated with HLJDD for 12 days. At the end of treatment, medium was removed and the cells were fixed with $4 \%$ paraformaldehyde in PBS and stained with $2 \%$ of crystal violet.

\subsection{Nascent protein synthesis assay}

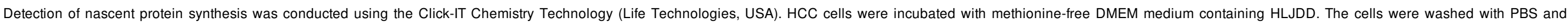

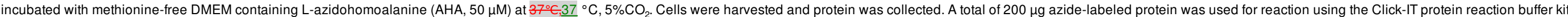

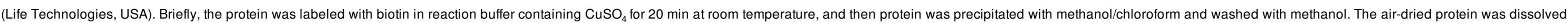
in RIPA buffer for further analysis.

\subsection{Immunoblotting}

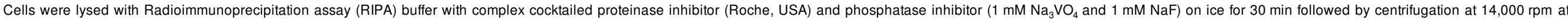

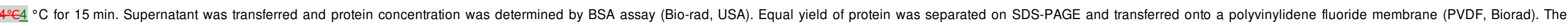

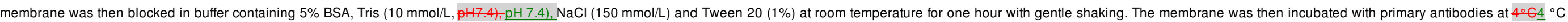

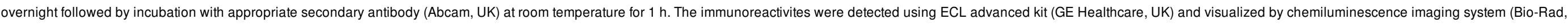
USA)

\subsection{Xenograft model}

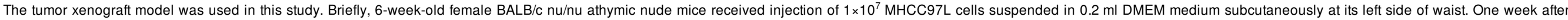

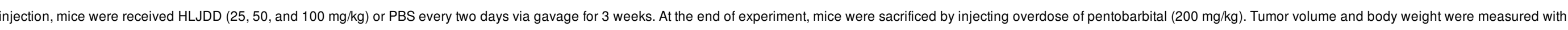

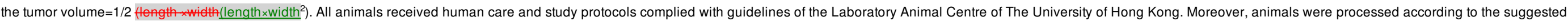
international ethical guidelines for the care of laboratory animals throughout the experiments.

\subsection{Immunohistochemistry}




\section{elsevier_JEP_9328}

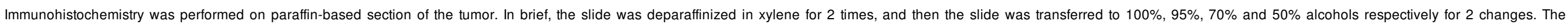

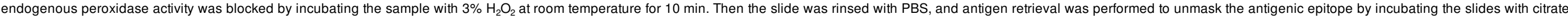

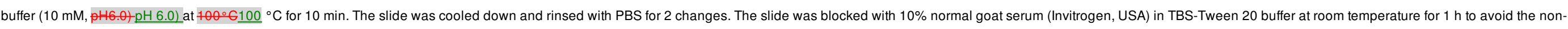

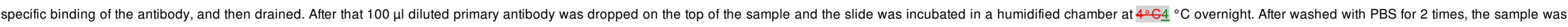

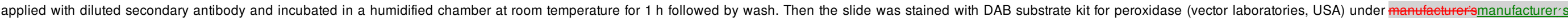

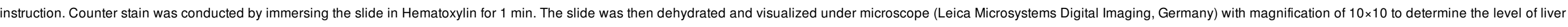
damage. Images were captured with Leica DFC 280 CCD camera.

\subsection{Statistical analysis}

All the studies were conducted in triplicate except particular notice. Data was expressed as mean $\pm \mathrm{SD}$. Statistical analysis was performed with Student $T$-Test.

\section{Results}

\subsection{Critical quality control on HLJDD}

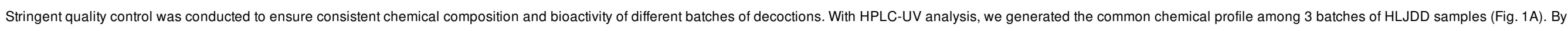

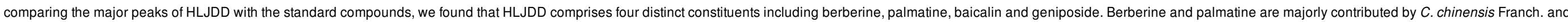

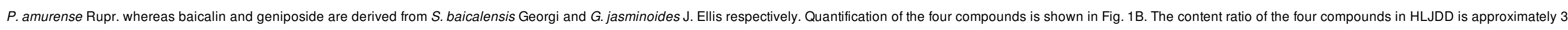
(geniposide, GEN): 3 (berberine, BBR): 1 (baicalin, BAl): 1 (palmatine, PAL). There are no harmful heavy metal and organic pesticides detected in all three batches (data not shown).

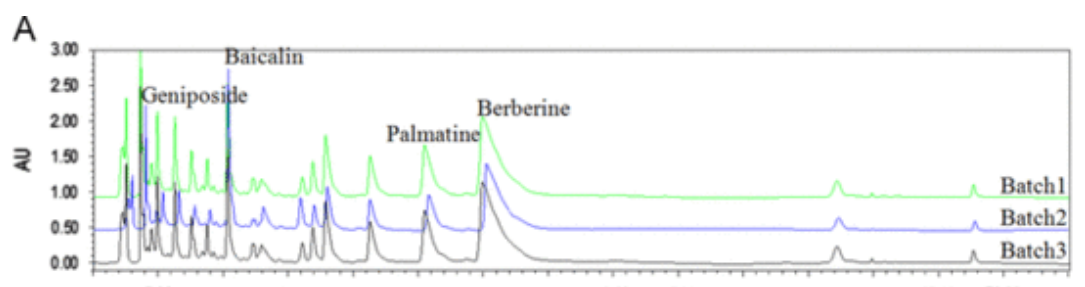

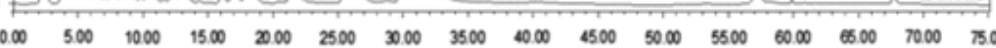
Mhutes

B

\begin{tabular}{ccc} 
Component & Retention time (min) & $\begin{array}{c}\text { Content }(\mathrm{mg} / \mathrm{g} \text { in } \\
\text { HLJDD) }\end{array}$ \\
\hline Geniposide & 5.04 & 23.5 \\
Baicalin & 9.88 & 7.5 \\
Palmatine & 24.07 & 8 \\
Berberine & 29.45 & 23.9 \\
\hline
\end{tabular}

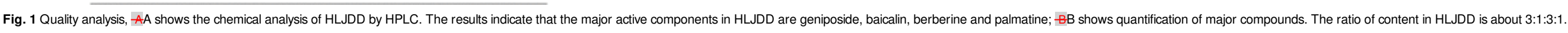

\subsection{HLJDD suppresses neoangiogenesis and xenografted growth of HCC}

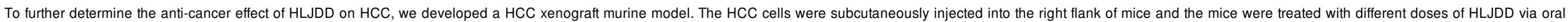

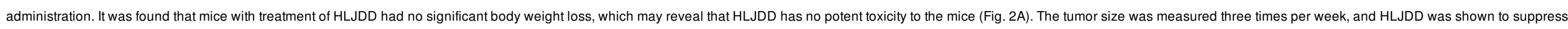

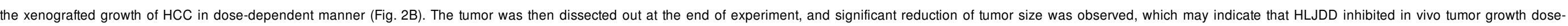

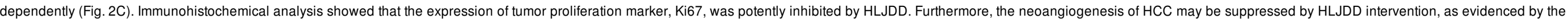

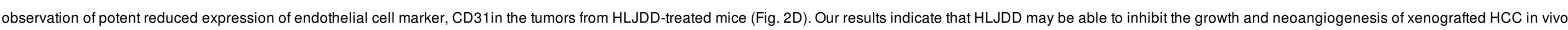




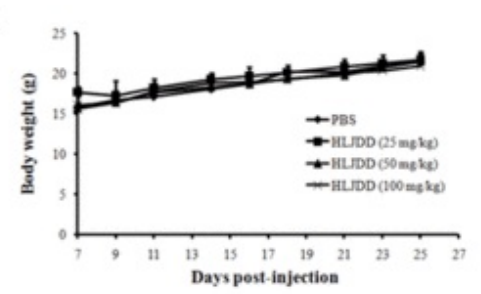

B

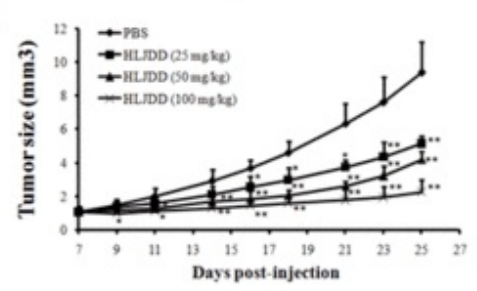

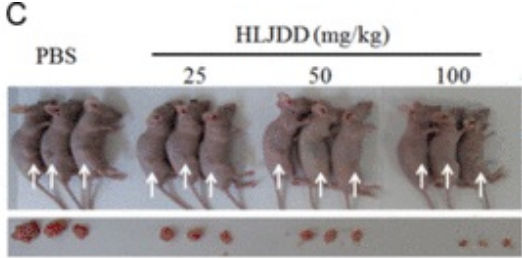

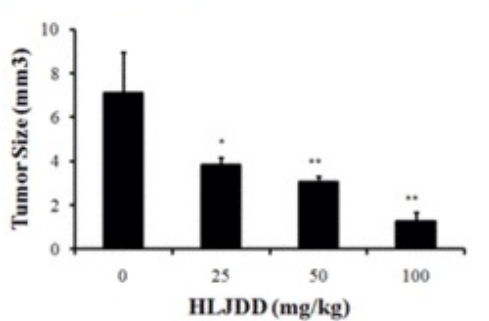

D

HIJDD (mg $/ \mathrm{kg}$ )

Ki67
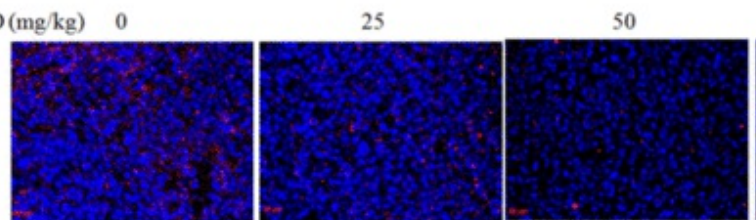

100

CD31
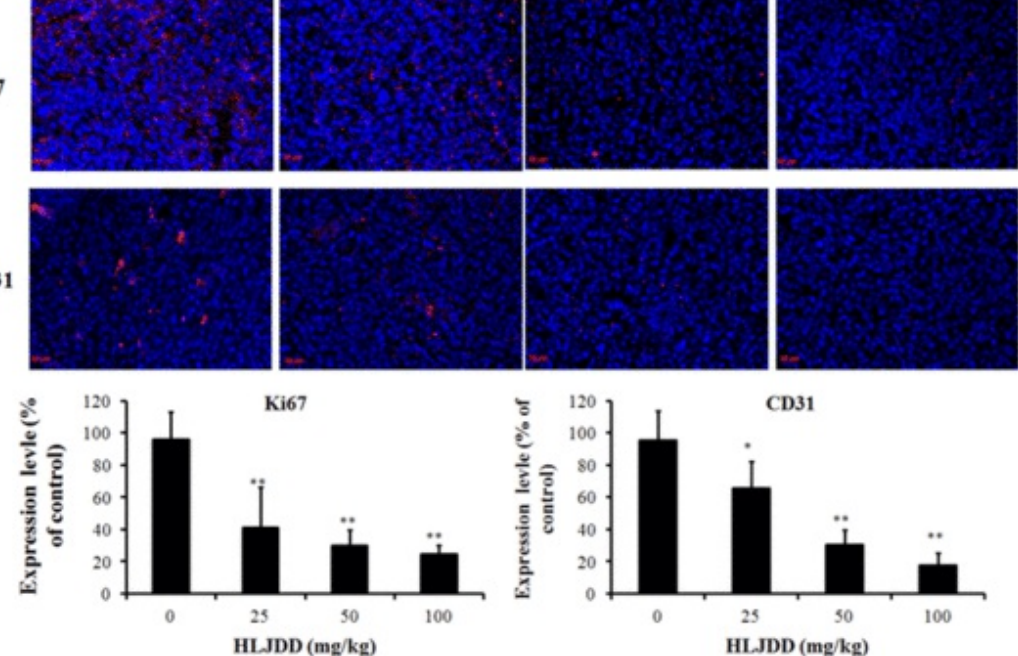

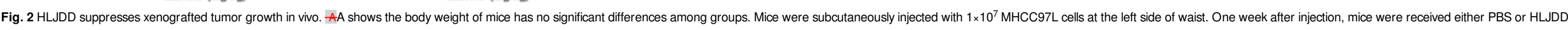

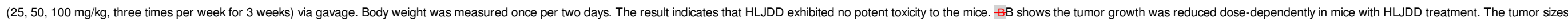

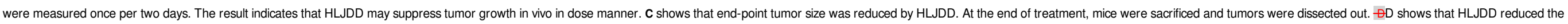

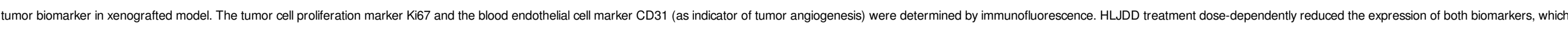
confirms the suppressive effect of HLJDD on in vivo tumor growth. ${ }^{*} p<0.05,{ }^{* *} p<0.01$ when compared to control.

\subsection{Nascent protein synthesis of HCC was blocked by HLJDD}

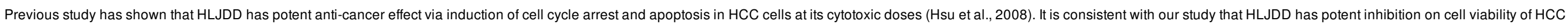

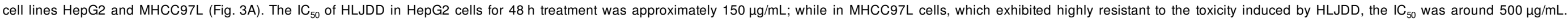

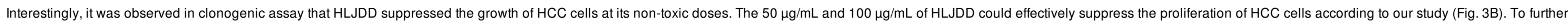




\section{elsevier_JEP_9328}

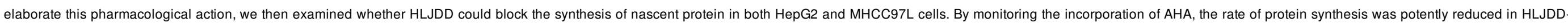

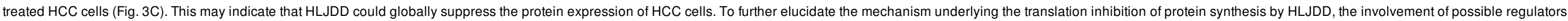
in translational control was determined. It was found that HLJDD has no significant effect on the expression and activity of protein translation initiation regulators, including elF4elF4A, elF4E and elF4G (Fig. 3D).
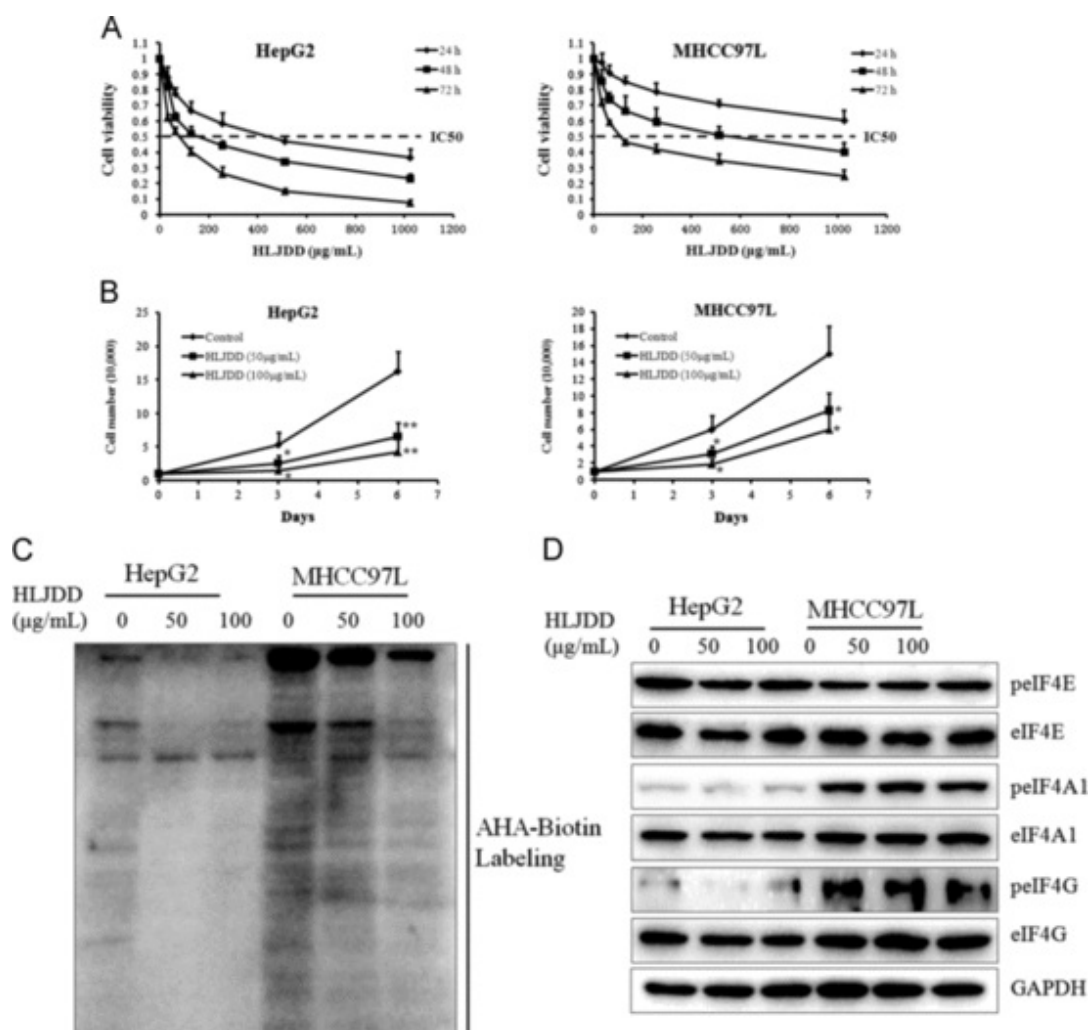

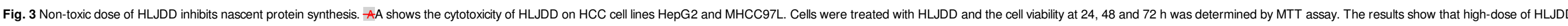

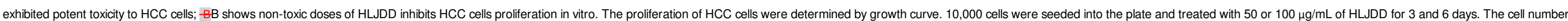

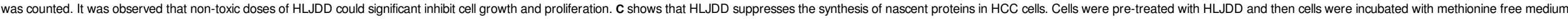

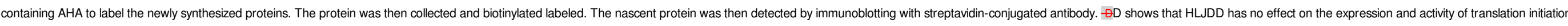
regulators.

\subsection{Components in HLJDD inhibit eEF2 activity in HCC cells}

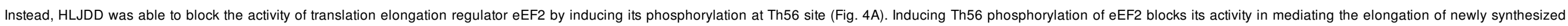

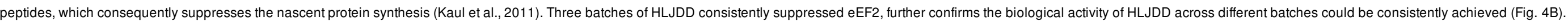

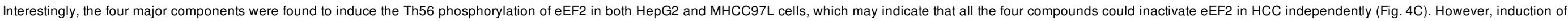

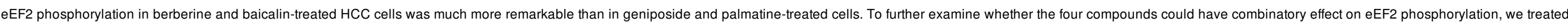

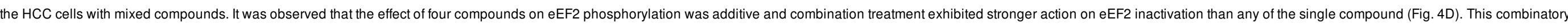

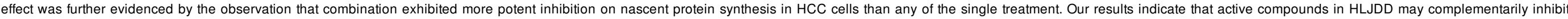




\section{elsevier_JEP_9328}

nascent protein synthesis in HCC cells via targeting on eEF2.

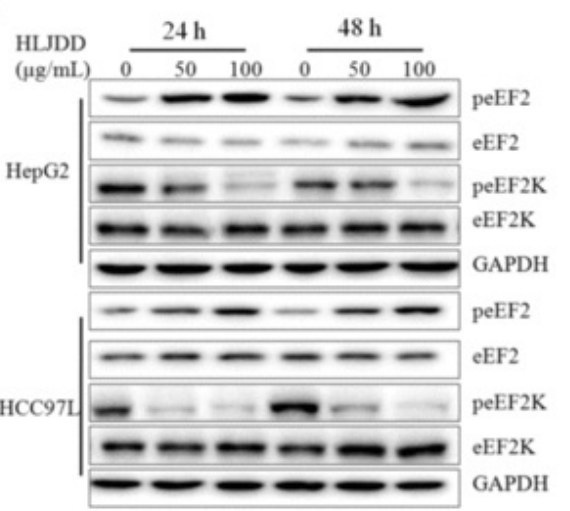

C

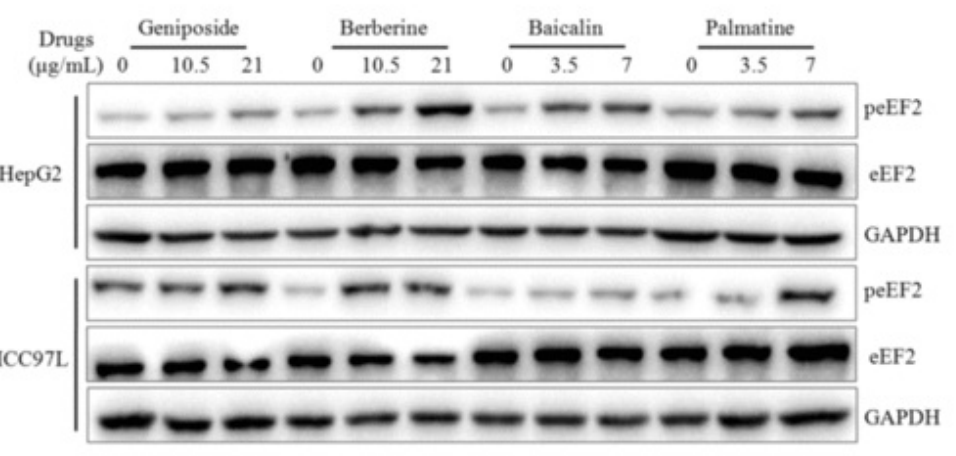

D

$\mathrm{HepG} 2$

MHCC97L

CTL GEN BBR BAI PAL GBBP CTL GEN BBR BAI PAL GBBP

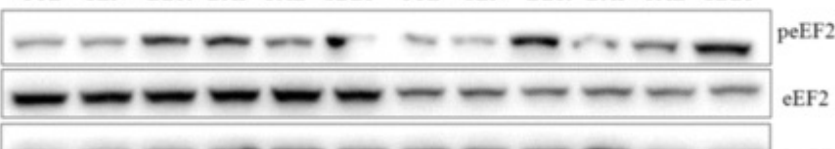

$---\infty-\infty-\infty \mathrm{GAPH}$

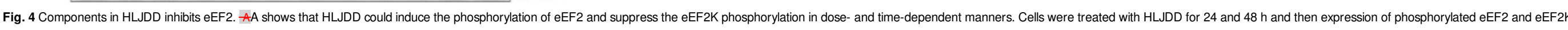

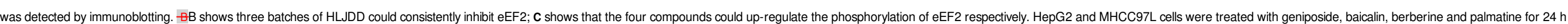
then collected. eEF2 phosphorylation was analyzed by immunoblotting. All the four compounds could induce phosphorylated eEF2 expression at different doses. ED shows that combination of the four compounds additively increases eEF2 phosphorylation.

\section{5 eEF2K/AMPK inhibition by HLJDD mediates eEF2 inactivation in HCC cells}

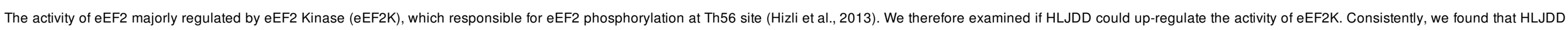

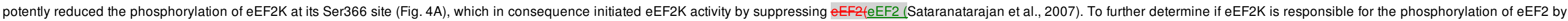

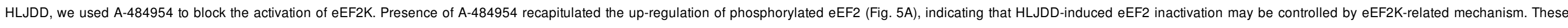

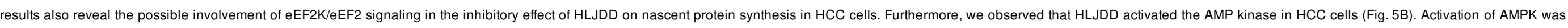

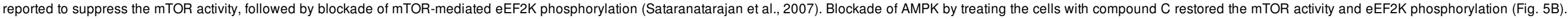

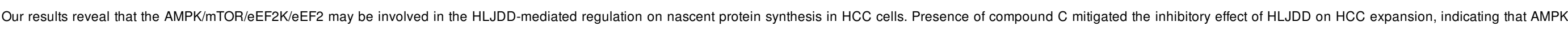




\section{elsevier_JEP_9328}

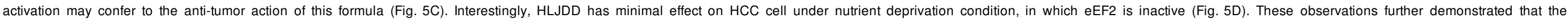
AMPK/eEF2K/eEF2 axis may contribute to the tumor inhibition by HLJDD in human HCC.
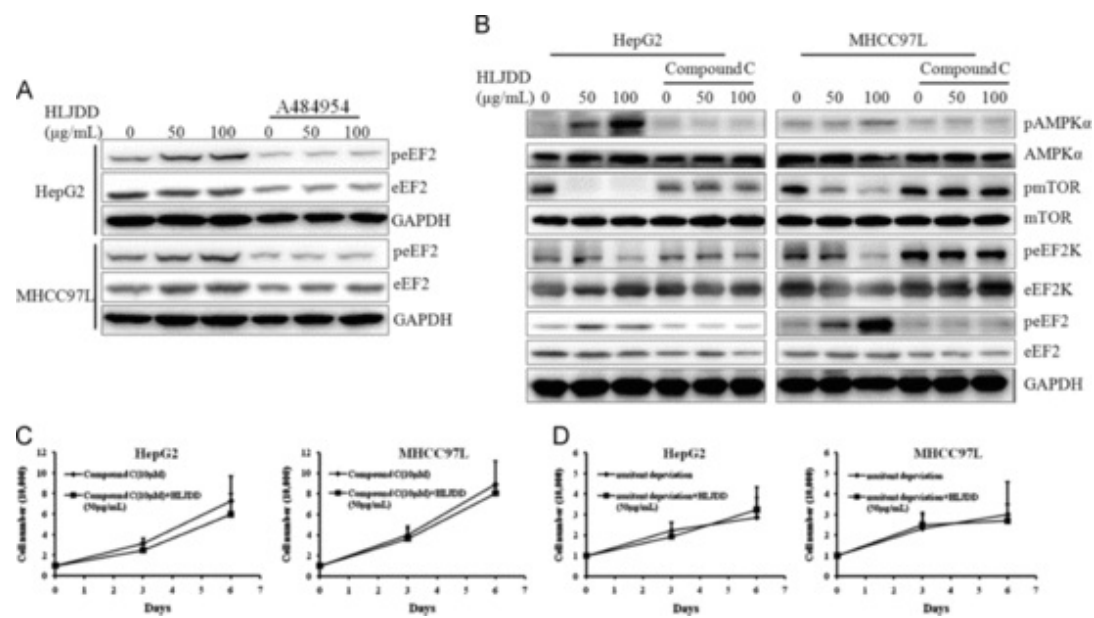

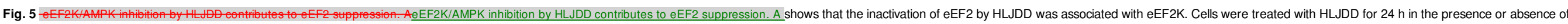

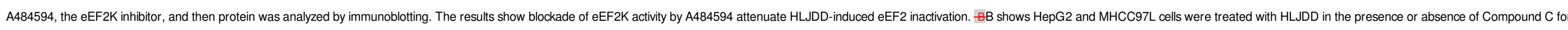

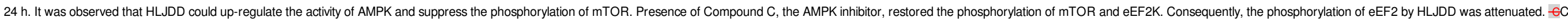

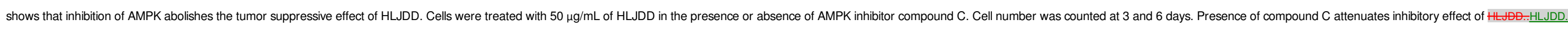

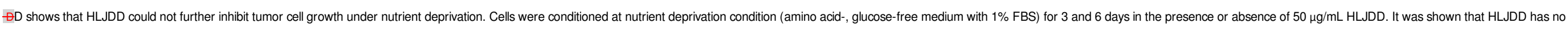
further inhibition on HCC under nutrient deprivation condition.

\section{Discussion}

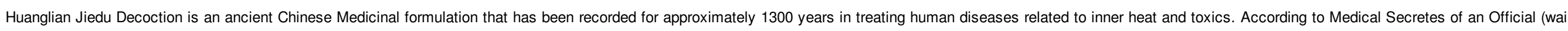

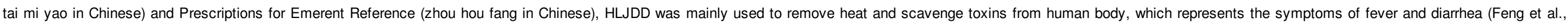

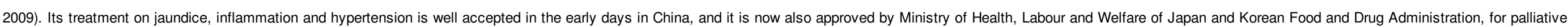

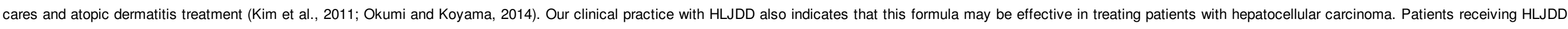

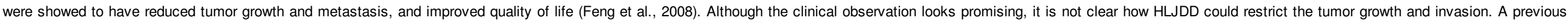

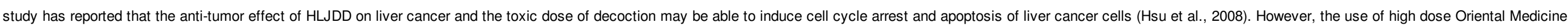

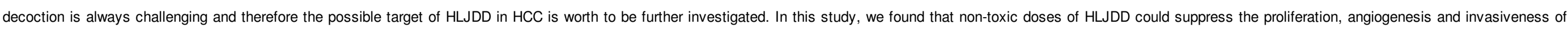

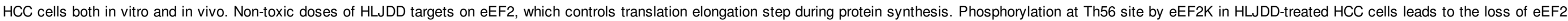

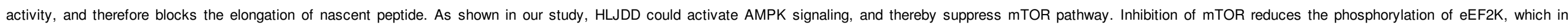

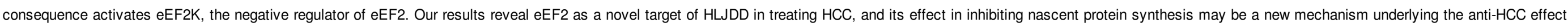
of HLJDD. The regulatory scheme underlying the anti-HCC action of HLJDD is shown in Fig. 6 . 


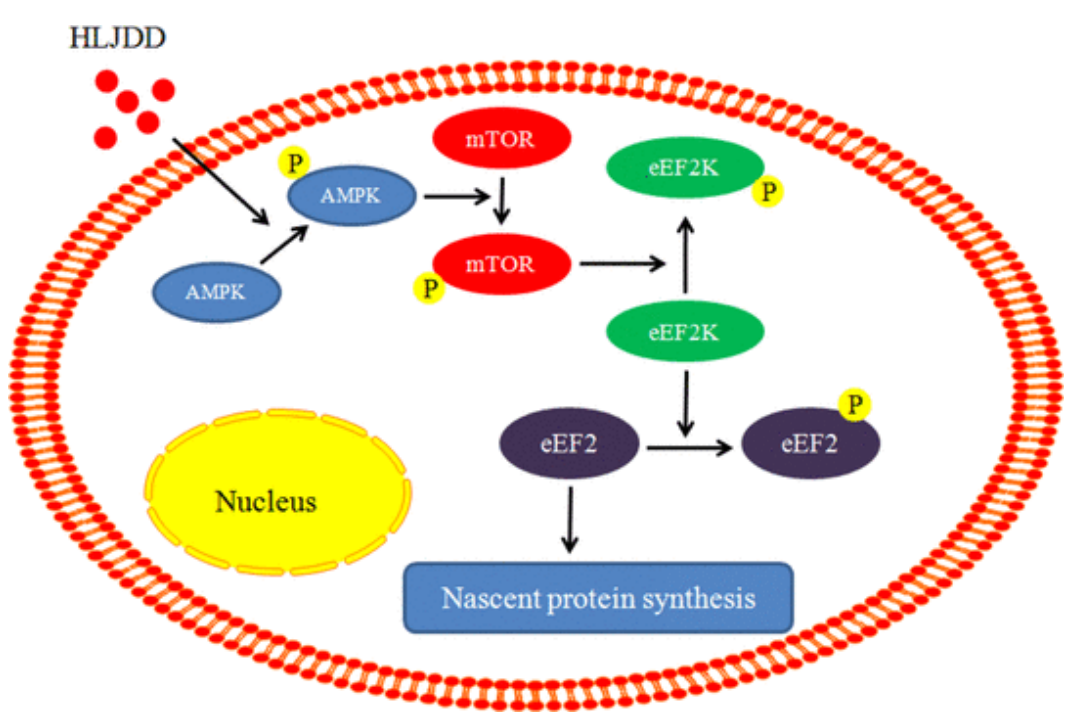

Fig. 6 Regulatory scheme of HLJDD on EEF2 activity in HCC.

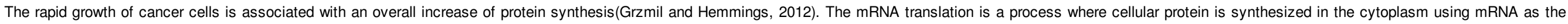

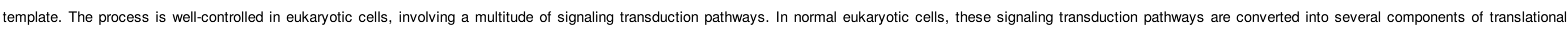

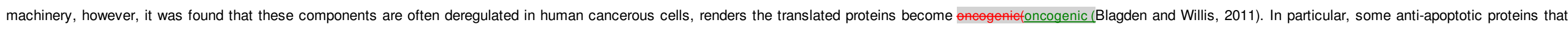

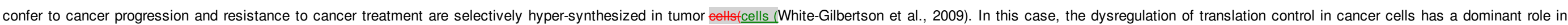

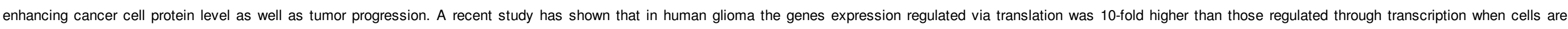

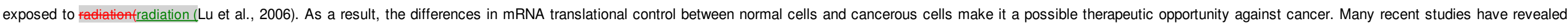

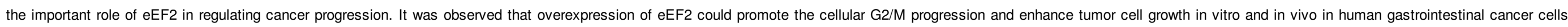

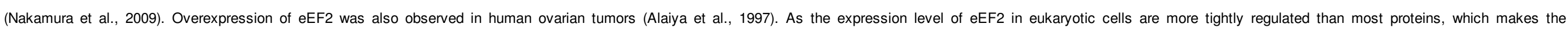

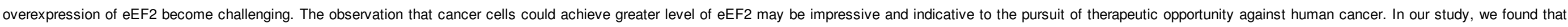

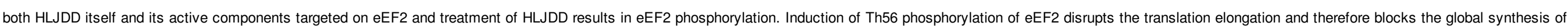

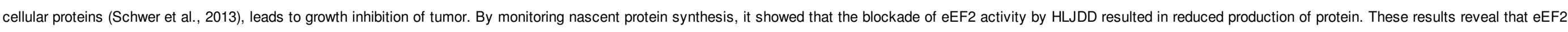

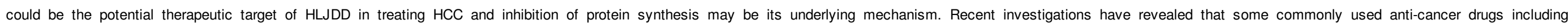

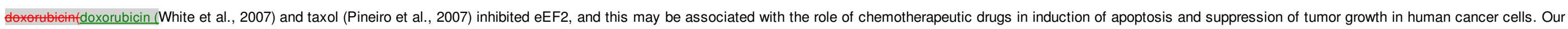
study may include HLJDD as a candidate in the list of complementary anti-HCC agents which target on eEF2.

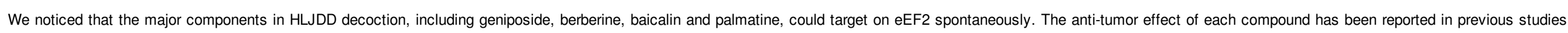

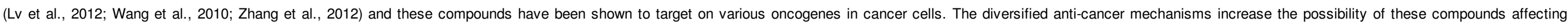

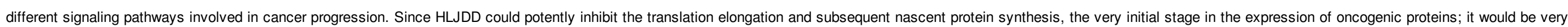

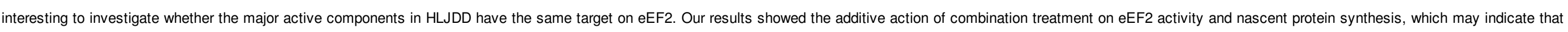

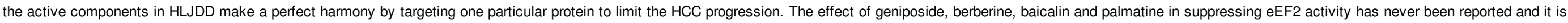

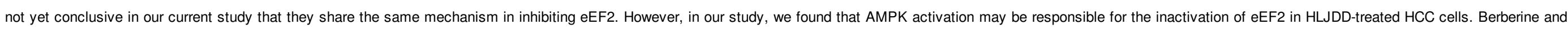

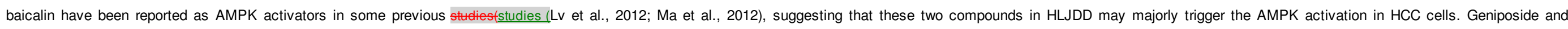

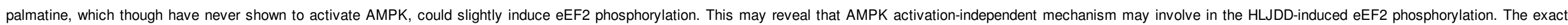




\section{elsevier_JEP_9328}

mechanism underlying the combinatory effect of active compounds in HLJDD needs further investigation.

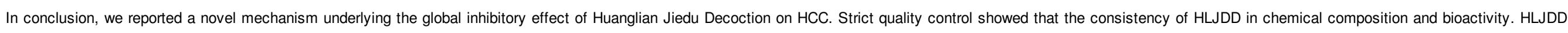

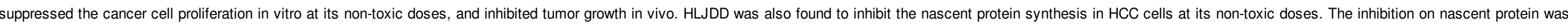

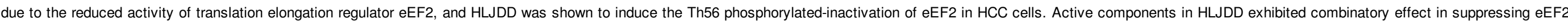

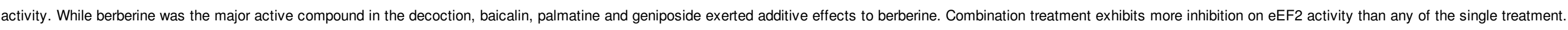

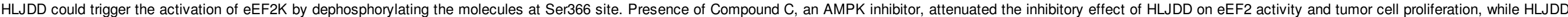

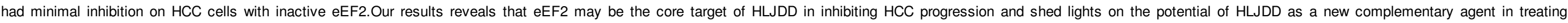
hepatocellular carcinoma.

\section{Conflict of Interestinterest}

The authors declare no conflict of interest for this study.

\section{AcknowledgementAcknowledgment}

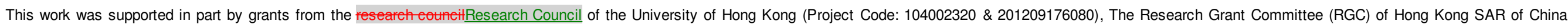

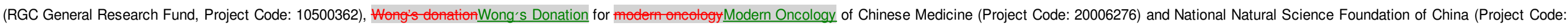

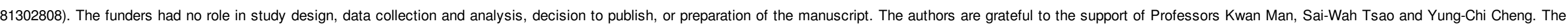
authors would like to express thanks to Ms Oi Yee Chow, Ms Cindy Lee, Mr. Keith Wong, and Mr. Freddy Tsang for their technical support.

\section{Reference}

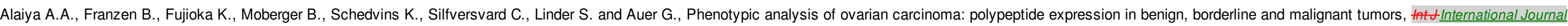
of Cancer 73, 1997, 678-683.

Blagden S.P. and Willis A.E., The biological and therapeutic relevance of mRNA translation in cancer., Nature Reviews Clinical oncology Oncology 8, 2011, 280-291.

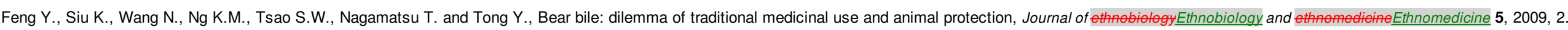

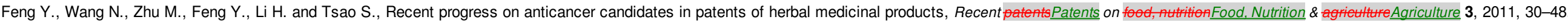

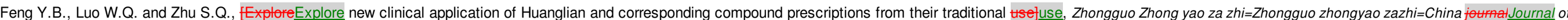
Chinese materia medica 33, 2008, 1221-1225.

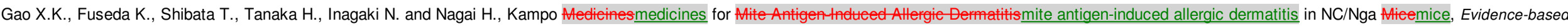
eomplementary and alternative Alternative Medicine: eCAM 2 2005, 191-199.

Ge. H., Tao. H. and Yang. Y., Bu fi zhou hou fangJi Zhou Hou Fang, 1996, Anhuianhui ke xue ji shu chu ban she; Hefei.

Grzmil M. and Hemmings B.A., Translation regulation as a therapeutic target in cancer, CancerResResearch 72, 2012, 3891-3900.

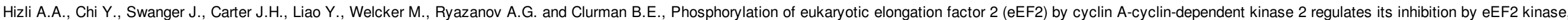
Molecular and eellutar biology Cellular Biology 33, 2013, 596-604.

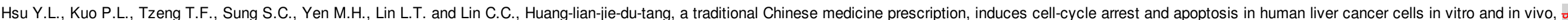
Gastrol Hepatolual of Gastroenterology and Hepatology 23, 2008, e290-299.

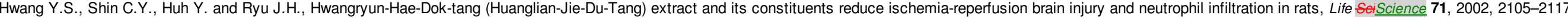




\section{elsevier_JEP_9328}

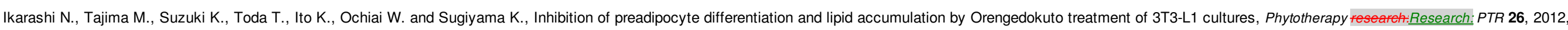
$91-100$.

Kaul G., Pattan G. and Rafeequi T., Eukaryotic elongation factor-2 (eEF2): its regulation and peptide chain elongation, Cell biochemistry and functionFunction 29, 2011, 227-234.

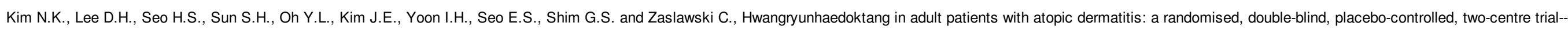
study protocol, BMC eomplementary Complementary and alternative Alternative Medicine 11, 2011, 68.

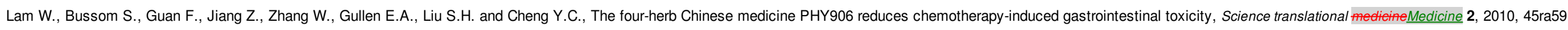

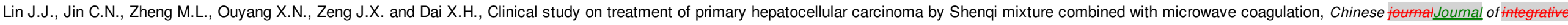
dicine Integrative Medicine 11, 2005, 104-110.

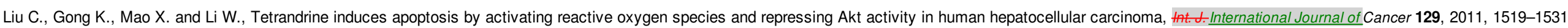

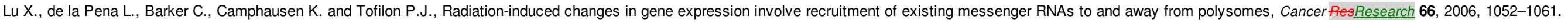

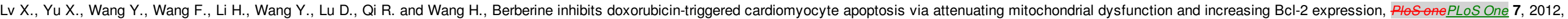
e47351.

Ma Y., Yang F., Wang Y., Du Z., Liu D., Guo H., Shen J. and Peng H., CaMKKbeta is involved in AMP-activated protein kinase activation by baicalin in LKB1 deficient cell lines, PloS onePLoS One 7, 2012, e47900.

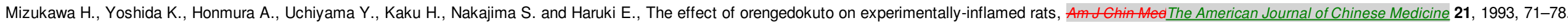

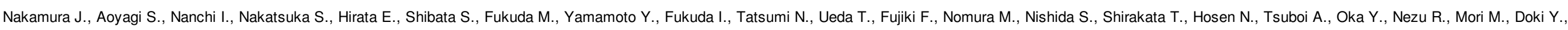

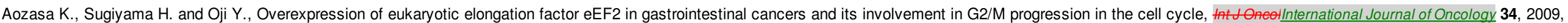
$1181-1189$.

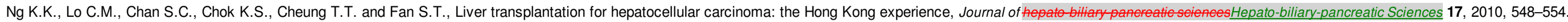

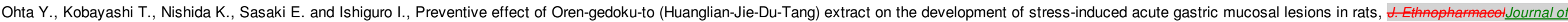
Ethnopharmacology 67, 1999, 377-384.

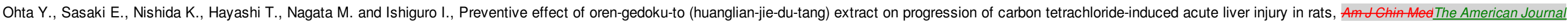
of Chinese Medicine 25, 1997, 57-68.

Okumi H. and Koyama A., Kampo medicine for palliative care in Japan, BioPsychoSocial medieineMedicine 8, 2014, 6.

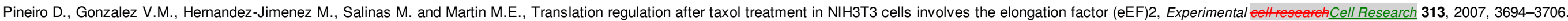

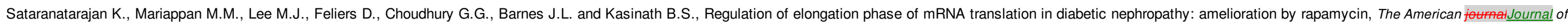
pathology 177 Pathology 171, 2007, 1733-1742.

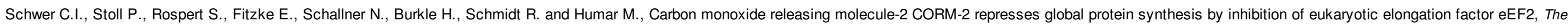
international journallinternational Journal of biomemistryiochemistry \& ell biology 45 Cell Biology 45. 2013, 201-212.

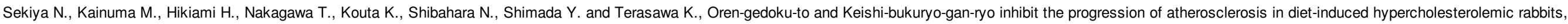
Biological \&

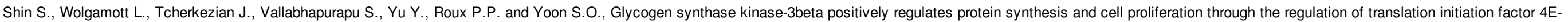




\section{elsevier_JEP_9328}

binding protein 1, Oncogene 33, 2014, 1690-1699.

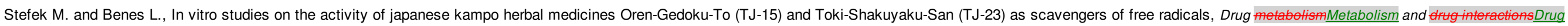
Interactions 11, 1994, 25-36.

Takase H., Imanishi K., Miura O., Yumioka E. and Watanabe H., Features of the anti-ulcer effects of Oren-gedoku-to (a traditional Chinese medicine) and its component herb drugs, Japanese journalJournal of Tharmacology Pharmacology 49, 1989, 301-308.

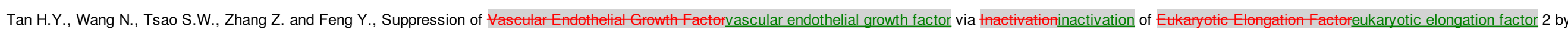
Alkaloidsalkaloids in Goptidiscoptidis rhizoma in Hepatocelltlar Gareinomahepatocellular carcinoma, Integrlntegrative Cancer Ther:Therapies, 2013.

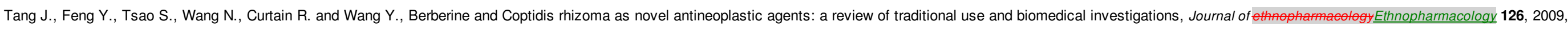
5-17.

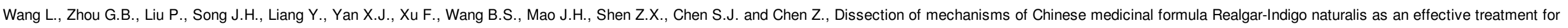
promyelocytic leukemia, Proceedings of the National Academy of Sciences of the United States of America 105, 2008, 4826-4831.

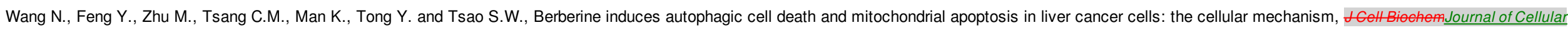
Biochemistry 111, 2010, 1426-1436.

Wang, T., 1955. Wai tai mi yao. Beijing: Ren min wei sheng chu ban she ying yin: Xin hua shu dian Beijing fa xing suo fa xing.

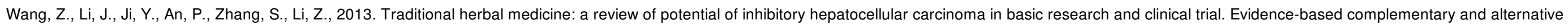
medicine: eCAM 2013, 268963.

White-Gilbertson S., Kurtz D.T. and Voelkel-Johnson C., The role of protein synthesis in cell cycling and cancer, Molecular

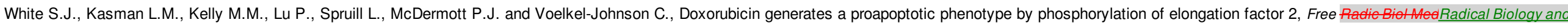
Medicine 43, 2007, 1313-1321.

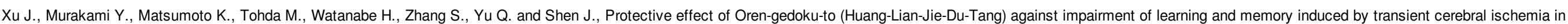
mice, JEthnopharmacolJournal of Ethnopharmacology 73, 2000, 405-413.

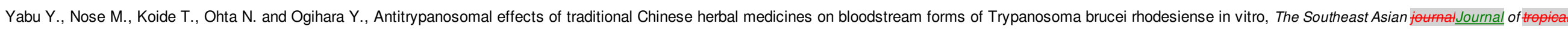
medicine Tropical Medicine and public health 29Public Health 29, 1998, 599-604.

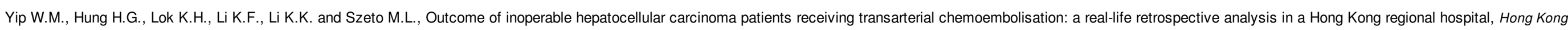
medical journal=Xianggang yi xue za zhi / Hong Kong Academy of Medicine 15, 2009, 339-345.

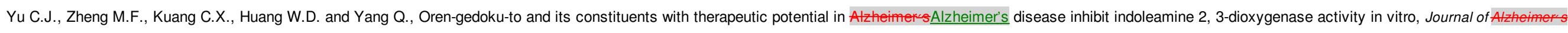
disease:Alzheimer's Disease: JAD 22, 2010, 257-266.

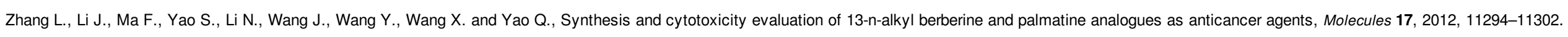

Zhou H. and Mineshita S., The effect of Oren-gedoku-to on experimental colitis in rats, The Journal of pharmacy Pharmacy and phacology 51Pharmacology 51, 1999, $1065-1074$.

\section{Graphical abstract}


elsevier_JEP_9328

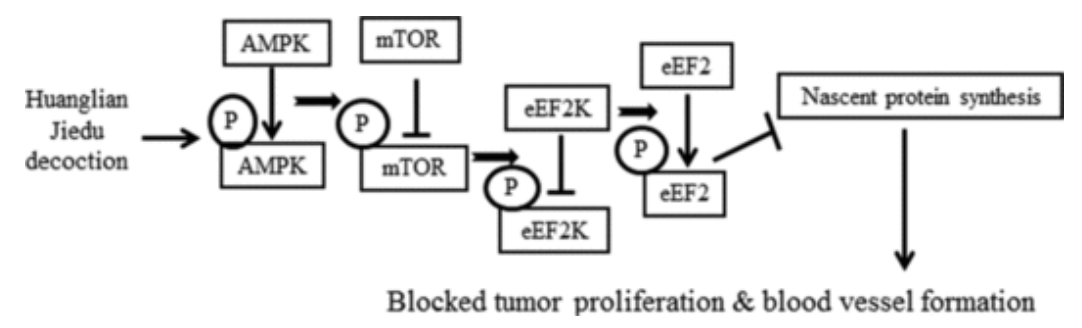

\section{Queries and Answers}

Query: Please confirm that given names and surnames have been identified correctly and are presented in the desired order.

Answer: Yes

Query: Please check the sponsor name and correct if necessary. "The Research Grant Committee (RGC) of Hong Kong SAR of China, Hong Kong". Answer: Yes

Query: Please check the sponsor name and correct if necessary. "National Natural Science Foundation of China, China".

Answer: Yes 